\title{
$\mathrm{Jill}^{\text {PAe }}$
}

\section{Inertial stretching separation in binary droplet collisions}

\author{
Karrar H. Al-Dirawi ${ }^{1}{ }^{\dagger}$, Khaled H.A. Al-Ghaithi ${ }^{2}$, Thomas C. Sykes ${ }^{2,3}$, \\ J. Rafael Castrejón-Pita ${ }^{4}$ and Andrew E. Bayly ${ }^{1, \dagger}$ \\ ${ }^{1}$ School of Chemical and Process Engineering, University of Leeds, Leeds LS2 9JT, UK \\ ${ }^{2}$ EPSRC Centre for Doctoral Training in Fluid Dynamics, University of Leeds, Leeds LS2 9JT, UK \\ ${ }^{3}$ Department of Engineering Science, University of Oxford, Oxford OX1 3PJ, UK \\ ${ }^{4}$ School of Engineering and Materials Science, Queen Mary University of London, London E1 4NS, UK
}

(Received 16 March 2021; revised 7 June 2021; accepted 19 July 2021)

\begin{abstract}
Binary droplet collisions exhibit a wide range of outcomes, including coalescence and stretching separation, with a transition between these two outcomes arising for high Weber numbers and impact parameters. Our experimental study elucidates the effect of viscosity on this transition, which we show exhibits inertial (viscosity-independent) behaviour over an order-of-magnitude-wide range of Ohnesorge numbers. That is, the transition is not always shifted towards higher impact parameters by increasing droplet viscosity, as it might be thought from the existing literature. Moreover, we provide compelling experimental evidence that stretching separation only arises if the length of the coalesced droplet exceeds a critical multiple of the original droplet diameters (3.35). Using this as a criterion, we provide a simple but robust model (without any arbitrarily chosen free parameters) to predict the coalescence/stretching-separation transition.
\end{abstract}

Key words: breakup/coalescence

\section{Introduction}

Binary droplet collisions exhibit a wide range of outcomes, including bouncing, permanent coalescence and eventual breakup (Ashgriz \& Poo 1990; Qian \& Law 1997; Pan et al. 2016, 2019). Understanding the underlying fluid physics of collision outcomes, and the transitions between them, is both of significant fundamental interest and tremendous practical importance. A canonical regime map showing the most common collision outcomes for equal-sized binary droplet collisions in terms of an impact parameter

$\dagger$ Email addresses for correspondence: k.h.n.al-dirawi@leeds.ac.uk, a.e.bayly@leeds.ac.uk 


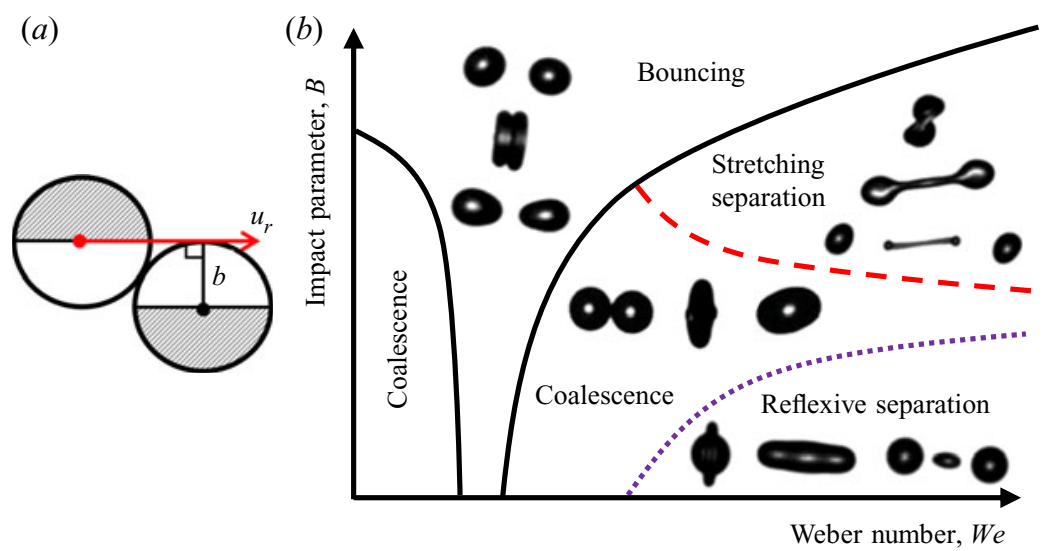

Figure 1. (a) Schematic of the geometry of a collision; the shaded regions indicate the non-interaction regions. (b) A typical regime map for binary droplet collisions, showing the most common collision outcomes. The C-SS transition (red dashed line) is of interest here (cf. figure 8); the C-reflexive separation (RS) transition (purple dotted line) is considered in $\S 3.4$.

$B=b / D_{o}$ (see figure $1 a$ ) and Weber number $W e=\rho D_{o} u_{r}^{2} / \sigma$ is shown in figure $1(b)$. Here, $\rho, D_{o}, u_{r}, \sigma$ and $b$ are the density, initial droplet diameter, relative velocity, surface tension and dimensional offset between the droplets (as shown in figure 1a), respectively. In this work, we are primarily interested in the transition from coalescence (C) to stretching separation (SS), which occurs when two equal-size droplets collide at sufficient $W e$ and $B$, as illustrated by the red dashed line in figure $1(b)$. In SS, the droplets temporarily coalesce and stretch by the non-interaction regions, before breaking up.

In many applications, such as nuclear reactors (Moretto et al. 1992), spray drying (Tsapis et al. 2005) and fuel sprays in combustion engines (Wang et al. 2005), uncontrolled collisions occur. Consequently, the particular collision outcome attained alters the droplet size distribution and hence affects the process performance. On the other hand, particular collision outcomes may be targeted, such as when studying chemical reaction kinetics (Lee et al. 2015; Davis et al. 2017) and in encapsulation (Planchette, Lorenceau \& Brenn 2010). In the former, temporary C (e.g. SS) is desired to ensure short mixing time for understanding intermediate states in reaction dynamics, whereas permanent $\mathrm{C}$ is generally required in the latter. Predicting the C-SS transition is therefore of both fundamental interest and practical importance.

Acquiring a complete description of the physics underpinning the C-SS transition is challenging owing to the various interconnected factors involved, including the offset distance, inertia and capillary and viscous forces. Different conditions, captured by $B$, We and the Ohnesorge number $\left(O h=\mu / \sqrt{\rho \sigma D_{o}}\right)$, can lead to different stretching dynamics, see the two extremes in figure 2(a,b): high impact We of low $O h$ droplets (high deformability) and low impact We of high $O h$ droplets (low deformability), respectively. The highly deformable collision shows significant flattening and rim development (see $t=0.87 \mathrm{~ms}$ ), whereas the slightly deformable case maintains an almost cylindrical shape.

Although the C-SS transition has been reported since the 1960s (e.g. Gunn 1965), the lack of comprehensive experimental measurements characterising the dynamics at this transition is a significant limitation of the literature. Previous studies have focused on experimentally defining the C-SS transition for nearly inviscid droplets (water) and providing phenomenological models by balancing inertia with the resisting 
(a)

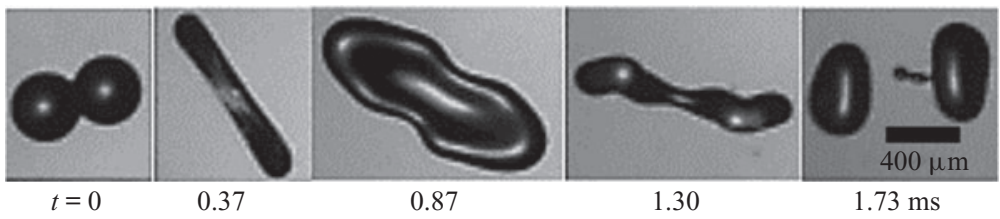

(b)

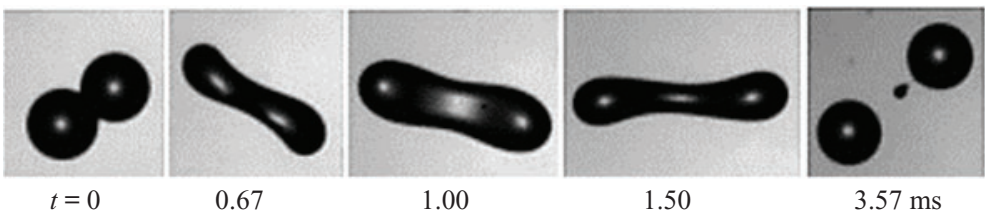

Figure 2. (a) Highly deformable SS, with $W e=90, O h=0.02$ and $B=0.37$. (b) Slightly deformable SS, with $W e=40, O h=0.16$ and $B=0.51$. Both cases in $(a)$ and $(b)$ capture the onset of SS.

capillary forces. However, the criterion of separation in this balance has always been a matter of debate with different authors and groups proposing different subjective criteria. Park (1970) suggested a criterion, which assumes that separation occurs only if the angular momentum exceeds the surface tension in the regions of the interaction. Brazier-Smith, Jennings \& Latham (1972) suggested a different criterion, which states that separation occurs only if the rotational energy is greater than the additional surface energy required to reform the initial droplets from the coalesced droplet. Another criterion was suggested by Arkhipov, Vasenin \& Trofimov (1983), which hypothesises that separation occurs only if the rotational energy, based on the angular velocity and the moment of inertia, is greater than the surface energy of the regions of non-interaction. Ashgriz \& Poo (1990) argued that the criteria of these models, which are based on the comparison of the rotational energy with surface energy, are not experimentally observed since separation happens before the development of any significant rotation. Therefore, Ashgriz \& Poo (1990) proposed a new criterion, which states that separation occurs only if the effective kinetic energy exceeds the surface energy in the regions of interaction. The effective kinetic energy is defined by the kinetic energy of the non-interaction regions in addition to that obtained from the regions of interactions using only the component of the drop velocities in the separating direction. Ashgriz \& Poo (1990) showed that their model performs better than the other models. Evidently, there is a need for an experimentally set criterion for the C-SS transition.

Since the 1990s (e.g. Jiang, Umemura \& Law 1992), the focus of the literature has shifted to the role of viscosity. In particular, it has been reported that, for a given We, increasing the droplet viscosity (i.e. Oh) shifts the C-SS transition to higher impact parameters (e.g. Jiang et al. 1992; Gotaas et al. 2007b; Planchette, Lorenceau \& Brenn 2012; Kuschel \& Sommerfeld 2013; Sommerfeld \& Kuschel 2016), that is, the red dashed line in figure $1(b)$ translates upwards. Some studies have developed viscous models to predict the C-SS transition by balancing inertial forces with the resisting capillary and viscous forces (Jiang et al. 1992; Pan et al. 2019; Suo \& Jia 2020). However, the viscous forces have always been treated by adjustable parameters subject to arbitrary choices, while the inertial and the regions of resistant capillary forces continued to be subjectively specified without consideration of the difference in the intermediate geometries that might arise from different combinations of $W e, B$ and $O h$, as seen in the second and third columns of figure 2.

In this paper, we uncover unique behaviours of the C-SS transition via extensive experimental measurements. Prominently, we show that this transition is inertial for a 


\section{K.H. Al-Dirawi and others}

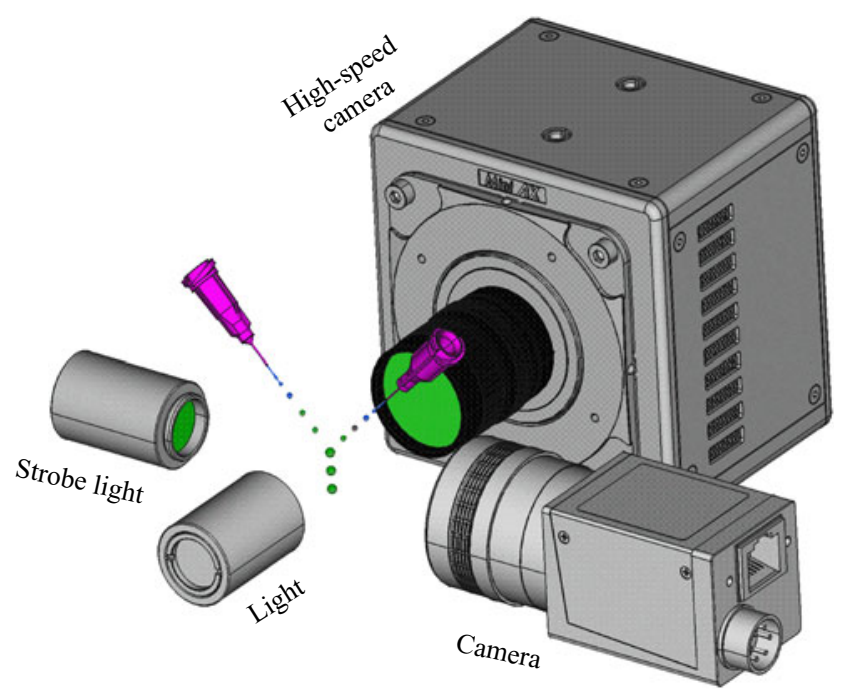

Figure 3. A sketch of our experimental set-up.

wide range of $O h$, where viscosity has no effect. Remarkably, a viscous effect exists for $O h$ values above the inertial range that we have identified. We also observe that SS occurs only if the stretched droplets reach a particular non-dimensional critical length, regardless of $W e, O h$ and $B$. We use this experimentally observed critical length to propose a simple but robust model for the C-SS transition, in which all numerical parameters are directly specified by readily obtainable experimental data (i.e. the model does not contain any arbitrarily chosen free parameters). The proposed model is shown to be consistent with both our experiments and previous experimental data for various fluids sourced from the literature.

\section{Experimental method}

Our experimental set-up and methodology are described in detail by Al-Dirawi \& Bayly (2019, 2020). Briefly, fluid was driven through custom-made monodisperse (110, 160 and $210 \mu \mathrm{m}$ ) nozzles to create a continuous jet; square-wave signals applied to piezos built into the nozzle caused the jet to breakup into a stream of equal-sized droplets. Two streams were created, which intersected at a tunable angle (enabling a varying $W e$ ) to engender equal-size binary droplet collisions. The collisions were imaged in two perpendicular vertical planes, as seen in figure 3. The nozzles were mounted on translation stages, which were adjusted to ensure that the droplets collided at the plane imaged by the front-view high-speed camera; alignment was ensured by strobe imaging using a standard CCD USB camera (labelled 'camera' in figure 3). The collision dynamics were recorded using a Photron AX200 high-speed camera at 30000 f.p.s., with a resolution in the range from 13 to $20 \mu \mathrm{m} \mathrm{px}^{-1}$ and a field of view of $256 \times 384$ pixels.

The fluids studied were deionised water, an $83 \%$ aqueous Glycerol solution and several aqueous solutions of hydroxypropyl methylcellulose (HPMC), with mass concentrations of $2 \%$ to $8 \%$. For these fluids, $\mu \in[1,85] \mathrm{mPa} \mathrm{s}$ and $\sigma \in[46,72] \mathrm{mN} \mathrm{m}^{-1}$, with $D_{o} \in[200,600] \mu \mathrm{m}$ and $O h \in[0.005,0.475]$ in our experiments. Solutions of HPMC show Newtonian rheology within the range of mass concentrations used in this work. 


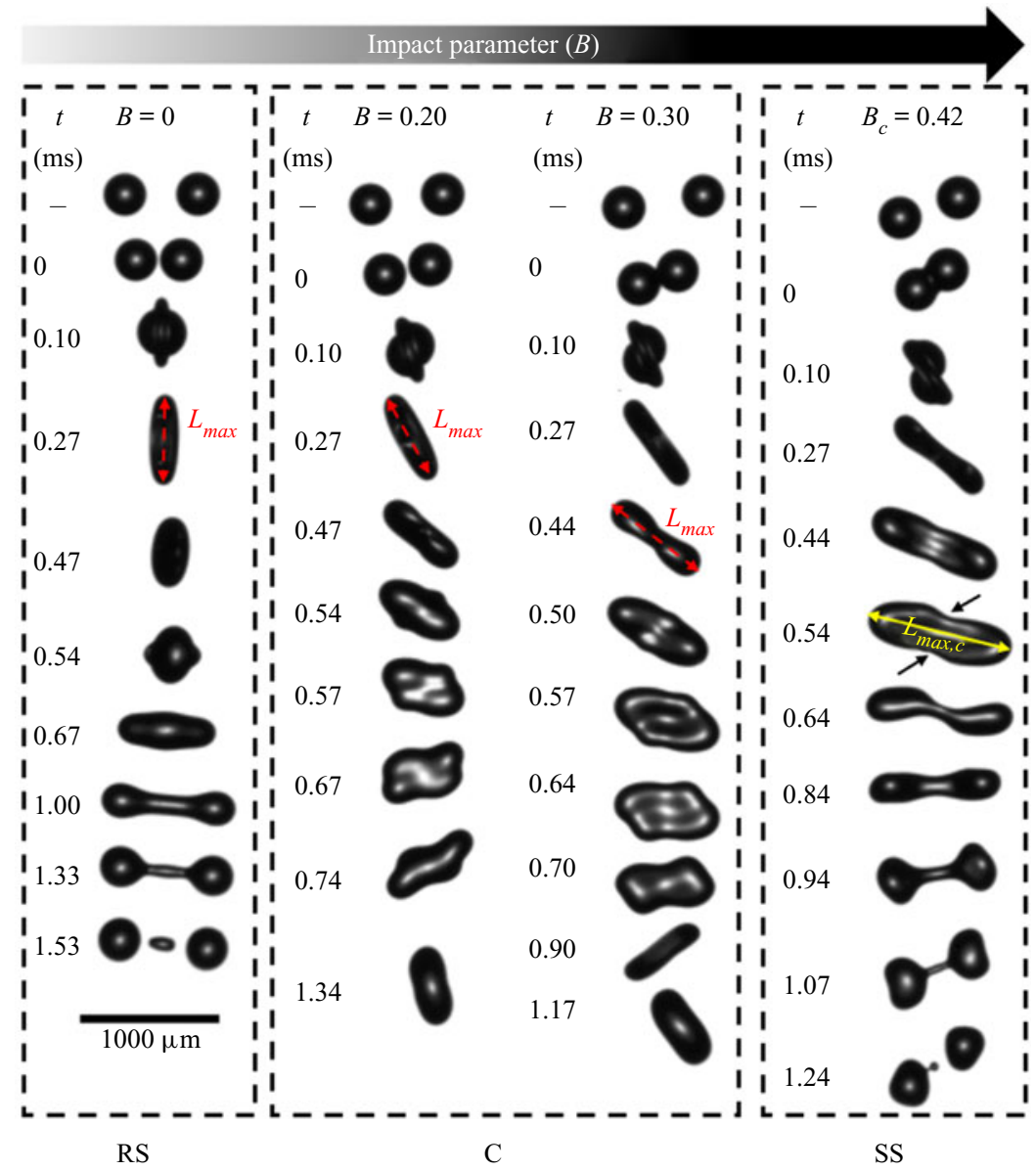

Figure 4. Dynamics of $2 \% \mathrm{HPMC}(\mathrm{Oh}=0.021)$ droplet collisions at $W e=56$ for different impact parameters $B$, from head-on collision exhibiting RS (first column), through $\mathrm{C}$ (second and third columns), to the onset of SS (fourth column). The latter transition (between the second/third and fourth columns) is of primary interest here.

The experiments cover wide ranges of $W e \in[20,140]$ and $B \in[0,1]$. The offset $B$ was varied by applying a frequency shift between the two streams of droplets, as explained by Gotaas et al. (2007a).

\section{Results}

\subsection{Collision dynamics}

For collisions below the critical impact parameter for SS $\left(B_{c}\right)$, the stretched-droplet system deforms to a maximum length $\left(L_{\max }\right)$ when all its kinetic energy has been converted into surface energy and viscous loss, before retracting, see the second and third columns in figure 4; quantitative measurements showing retraction are provided (see supplementary material available at https://doi.org/10.1017/jfm.2021.674). Note that the first column in figure 4 is an example of RS, which occurs for much lower impact parameters than SS, as seen in figure $1(b)$. Indeed, given sufficiently large impact parameters (i.e. $B \geq B_{c}$ ), the 


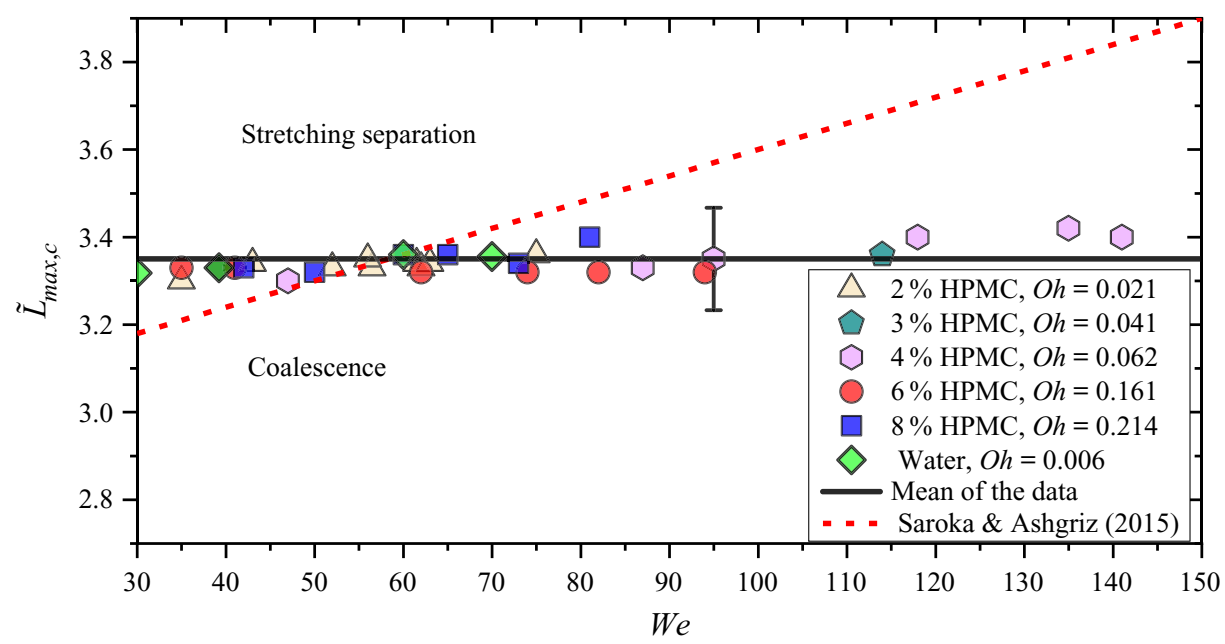

Figure 5. Critical maximum length leading to separation in terms of $W e$ and $O h$, for various $B_{c} \in[0.55,0.3]$. The error bar represents the propagation of the measurement uncertainty arising from the resolution (note that all points have the same order of error). The fit of Saroka \& Ashgriz (2015), $\tilde{L}_{\max , c}=3+0.006 \mathrm{We}$, is included for comparison.

non-interaction regions (see figure $1 a$ ) are large enough that the stretching energy (mainly the kinetic energy of the non-interaction regions) leads to SS, as seen in the last column of figure 4 (Ashgriz \& Poo 1990; Pan et al. 2019).

\subsection{Critical length for SS}

For $B>B_{c}$, SS is achieved by a pinching process that becomes significant after the stretched-droplet system realises its maximum length $\left(L_{\max , c}\right)$, see the last column of figure 4 at $t \geq 0.54 \mathrm{~ms}$. The dynamics after maximum stretching is similar to those of ligament breakup. In cylindrical ligaments, breakup or retraction to equilibrium depends on the aspect ratio, the critical value of which depends in turn on Oh (Castrejón-Pita, Castrejón-Pita \& Hutchings 2012). Similarly, a transition between C and SS is expected if the system, at the maximum stretching, achieves a certain critical aspect ratio. Owing to the collision dynamics, the stretched droplet is not quite cylindrical at the instant of $L_{\text {max }, c}$, so a normalised critical length $\left(\tilde{L}_{\max , c}=L_{\max , c} / D_{o}\right)$ is used here in place of the aspect ratio. Remarkably, our experiments for all $O h \in[0.005,0.475]$ and $W e \in[20,140]$ investigated reveal that $\tilde{L}_{\text {max }, c} \approx 3.35$, regardless of $B_{c}$, as shown in figure 5. Note that recent numerical work has suggested that this normalised critical length is a function of the Weber number, which was empirically determined (by fitting) to be $\tilde{L}_{\max , c}=3+0.006 \mathrm{We}$ (Saroka \& Ashgriz 2015). This fit was based on relatively narrow range of data, for $O h<6.7 \times 10^{-3}$, We $\in[20,50]$ and $B \in[0.95,0.5]$. It is also plotted (red dashed line) in figure 5, from which it can be seen to be inconsistent with our experimental data (note the error bar displayed), especially at high We values.

It is interesting to compare our constant critical length for SS with critical aspect ratios of liquid ligaments reported in the literature. To allow for this comparison, an aspect ratio equivalent to our critical length needs to be determined. Based on volume conservation for a cylinder with $\tilde{L}_{\max , c}=3.35$, we calculated our equivalent aspect ratio as 5.13. However, reported critical aspect ratios of cylindrical ligaments within the range of $\mathrm{Oh}$ 
investigated in this work are of the order of 20 (figure 5 in Castrejón-Pita et al. 2012). This significant difference may be attributable to the initial shape of the collision-generated ligament (at maximum deformation), which has an initial contraction resulting from the geometry of the off-centred collision, see the arrows in the last column of figure 4 at $t=0.54 \mathrm{~ms}$. Planchette et al. (2017) reported a significantly smaller critical aspect ratio of approximately 3 for cylindrical ligaments with $O h \leq 0.1$, resulting from the RS of droplets colliding head-on (first column of figure 4). This small aspect ratio may be due to the presence of a reflexive flow induced by the retraction of a rimmed-lamellar disk, which assists the pinch-off at the centre and delays the retraction of the ends of the formed cylinder. Note that comparisons with aspect ratios of liquid column breakup owing to the Rayleigh instability, which suggest a critical aspect ratio equal to $\pi$ (Rayleigh 1878), and of the linear instability of liquid threads being pinched from a nozzle (Henderson et al. 2000; Dong, Carr \& Morris 2006), are not appropriate owing to the different geometries involved. In the former, there are no rounded ends that lead to retraction; in the latter, there is only one rounded end that leads to retraction of the thread. The comparisons made here reveal that SS shows a unique equivalent critical aspect ratio.

\subsection{Model for the C-SS transition}

The normalised critical length, $\tilde{L}_{\max , c}$, can be used as a criterion to predict the C-SS transition $B_{c}(W e, O h)$, given that obtaining $\tilde{L}_{\max }(B, W e, O h)$ is feasible. Figure $6(a)$ presents $\tilde{L}_{\max }$ as a function of $B$ for various combinations of We and $O h$. Remarkably, for all cases regardless of $W e$ and $O h$, two distinct behaviours are seen for $\tilde{L}_{\text {max }}$, depending on $B$ : (I) $\tilde{L}_{\max }$ remains constant for $B \leq 0.2$ with a value equal to $\left.\tilde{L}_{\max }\right|_{B=0}$; and (II) a region of linear proportionality for $B>0.2$ and slightly greater than $B_{c}$. Given these two behaviours, we can write $\left.\tilde{L}_{\max }\right|_{B>0.2}=a+s B$, where $a=\left.\tilde{L}_{\max }\right|_{B=0}-0.2 s$; $a$ and $s$ are the intercept and the slope of the linear fits in region II, respectively. At $B=B_{c}$, $\left.\tilde{L}_{\text {max }}\right|_{B>0}=\tilde{L}_{\text {max }, c} \approx 3.35$. Therefore, the C-SS transition is given by

$$
B_{c}=\frac{3.35-\left.\tilde{L}_{\max }\right|_{B=0}+0.2 s}{s} .
$$

However, as can be seen from figure $6(a)$, the values of $\left.\tilde{L}_{\max }\right|_{B=0}$ and $s$ depend on $W e$ and Oh. Thus, in order to use (3.1) for predicting the C-SS transition as a function of We and $O h,\left.\tilde{L}_{\max }\right|_{B=0}$ and $s$ need to be quantified as a function of We and $O h$.

Quantifying $\left.\tilde{L}_{\max }\right|_{B=0}$ as a function of We and $O h$ can be achieved via an energy balance in the compression phase, between the instant of collision and the instant of maximum deformation ( $t=0.27 \mathrm{~ms}$ in the first column of figure 4$)$. The energy balance is accomplished assuming a pancake shape at the maximum deformation for head-on collisions with a diameter equal to $\left.\tilde{L}_{\max }\right|_{B=0}$ (as proposed by Willis \& Orme 2003). Hence, $\left.\tilde{L}_{\max }\right|_{B=0}$ is given by

$$
\left.\tilde{L}_{\max }\right|_{B=0}=\sqrt{\frac{(1-\alpha) W e+48}{12\left(1+\frac{8}{\left.3 \tilde{L}_{\text {max }}^{3}\right|_{B=0}}\right)}} .
$$

Here, $\alpha$ is the ratio of the viscously lost energy in the compression phase to the initial kinetic energy. This ratio was evaluated using experimental measurements in an energy 


\section{K.H. Al-Dirawi and others}
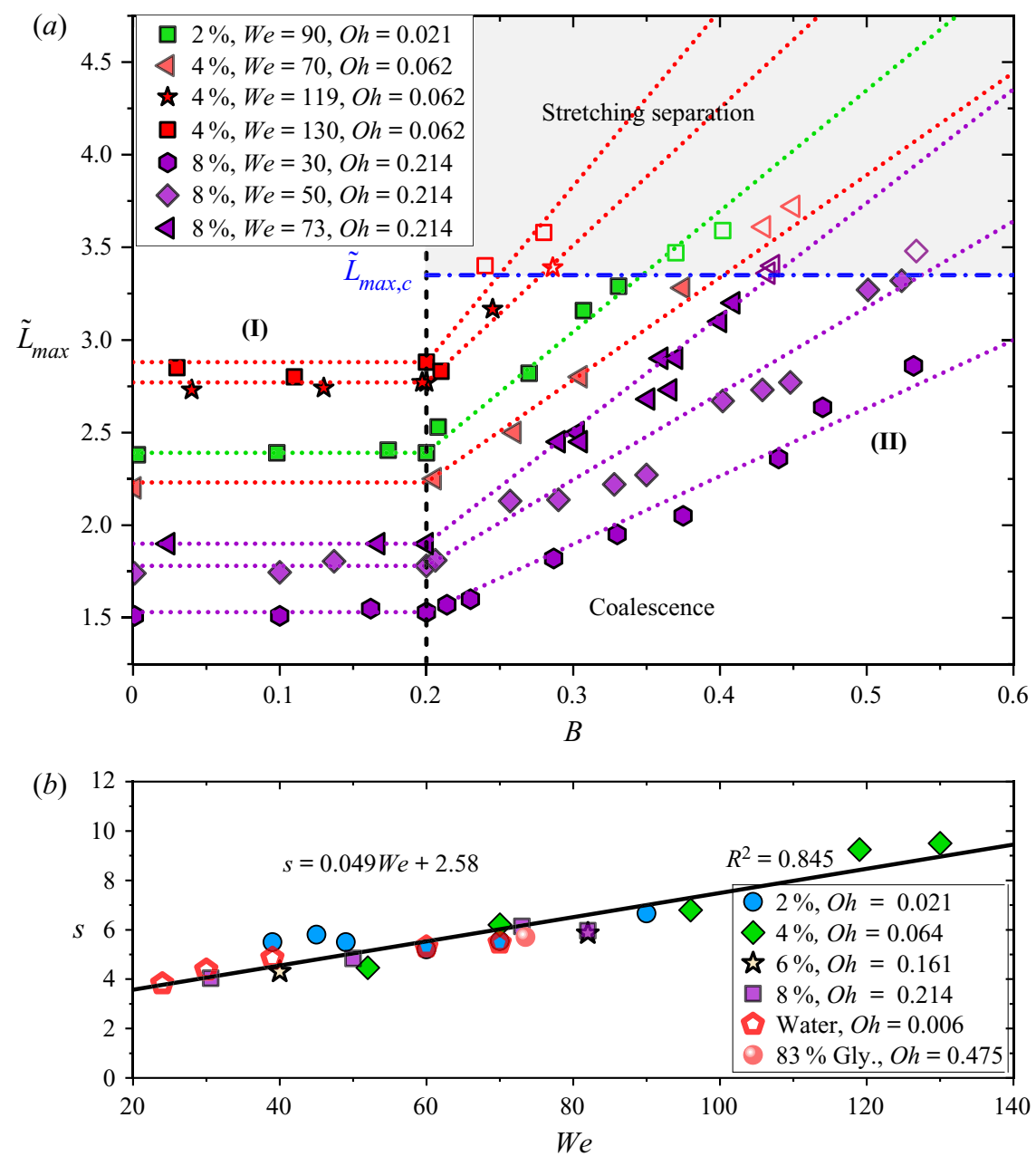

Figure 6. (a) Influence of the impact parameter, $B$ on the maximum length that the coalesced droplets attain, for a wide range of We and $O h$ (solid symbols, C, and hollow, SS). (b) The change in slope of $\tilde{L}_{\text {max }}(B)$ with We for $B>0.2$ up to the threshold of SS, for a wide range of $O h$.

balance by Planchette et al. (2017). Based on their extensive experimental measurements, and confirmed by measurements in this work, there are three regimes for $\alpha$ depending on $O h$, as shown in figure 7. At $O h \lesssim 0.02, \alpha$ generally increases with $O h$ from 0.50 to $0.65 \pm 0.05$. For $O h \in[0.02,0.14], \alpha$ remains constant at $0.65 \pm 0.05$, therefore exhibiting 'inertial behaviour' (independent of viscosity). For $\mathrm{Oh}>0.14$, the value of $\alpha$ varies greatly, but is generally above 0.65 . This behaviour of $\alpha$ is confirmed by our experiments. Further technical details (including the derivations of (3.2)) are provided as supplementary material.

Regarding quantifying $s$ as a function of We and $O h$, figure 6(b) shows $s$ versus $W e$ for a wide range of $O h$. The slope linearly increases with $W e$, while $O h$ has a negligible effect, given that all data collapse on to a single line. Therefore, for any combination of We and $O h, s$ can be described using a linear fit to the data in figure $6(b)$,

$$
s=0.049 W e+2.58 \text {. }
$$




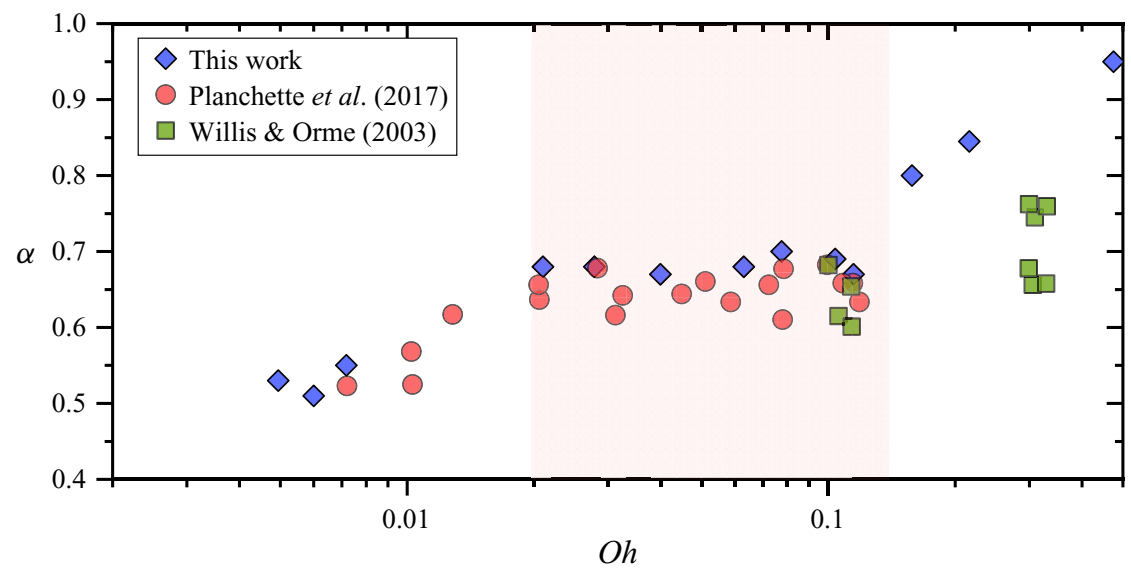

Figure 7. The measured ratio of viscous loss, in the compression phase at $B=0$, to the initial kinetic energy for a wide range of $\mathrm{Oh}$.

Estimating $\left.\tilde{L}_{\max }\right|_{B=0}(W e, O h)$ from (3.2), and using (3.3) enables the C-SS transition to be determined from (3.1), yielding a model that depends only on $O h$ via $\alpha$. As the latter is constant ( $\alpha=0.65 \pm 0.05$ ) for an order-of-magnitude-wide range of $O h$, from 0.02 to 0.14 , the model suggests that SS shows pure inertial behaviour within this wide range of $O h$. Therefore, the $\mathrm{C}-\mathrm{SS}$ transition is fixed over this range of $\mathrm{Oh}$. It is important to appreciate that all numerical values in the proposed model are not subject to arbitrary choices, but are instead directly specified by the experimental data, via relatively simple measurements. In particular, the model contains no arbitrarily chosen free fitting parameters.

Figure 8 shows all the available experimental data, both from our experiments and the literature, in terms of $W e$. As seen, all data within the inertial range collapse on to the transition predicted by the model using $\alpha=0.65$. It should be noted that this fixed transition is slightly higher in terms of $B$ than the transition of near-inviscid (e.g. water) droplets, as can be seen in the inset figure. However, when using the appropriate value of $\alpha$ for water (which is 0.5 , as specified by figure 7 ), the model is still able to accurately predict the near-inviscid C-SS transition. Here, it is important to mention that the frequently used model of Ashgriz \& Poo (1990) fails to accurately predict the transition for water as it underestimates $B_{c}$, as shown in the inset figure. This underestimation was also noted in numerical work by the original author in Saroka \& Ashgriz (2015). Overall, the small difference between the transition of water and the fixed (inertial) transition of $\alpha=0.65$ might indicate an inertial transition for a wider range of $O h \in[0.006,0.140]$.

For collisions of droplets with $O h>0.14$, the transition moves towards higher impact parameters, consistent with the behaviour reported in the literature (Gotaas et al. 2007b; Kuschel \& Sommerfeld 2013; Sommerfeld \& Kuschel 2016). For example, the data for $8 \%$ HPMC $(O h=0.214)$ shows a higher transition than observed for the inertial case. Using the data of Sommerfeld \& Pasternak (2019), sunflower oil $(O h=0.475)$ produces an even higher boundary in terms of $B$, as seen inset in figure 8 . Note that the proposed model remains valid for $O h>0.14$ when an appropriate $\alpha$ is used. For example, using $\alpha=0.85$ for $8 \%$ HPMC and $\alpha=0.97$ for sunflower oil (not reported in Sommerfeld \& Pasternak 2019) show excellent agreement. Here, $\alpha=0.97$ for sunflower oil was measured from experiments that were designed to match the relevant $O h=0.475$, using $450 \mu \mathrm{m}$ droplets of an $83 \%$ aqueous Glycerol solution. 


\section{K.H. Al-Dirawi and others}

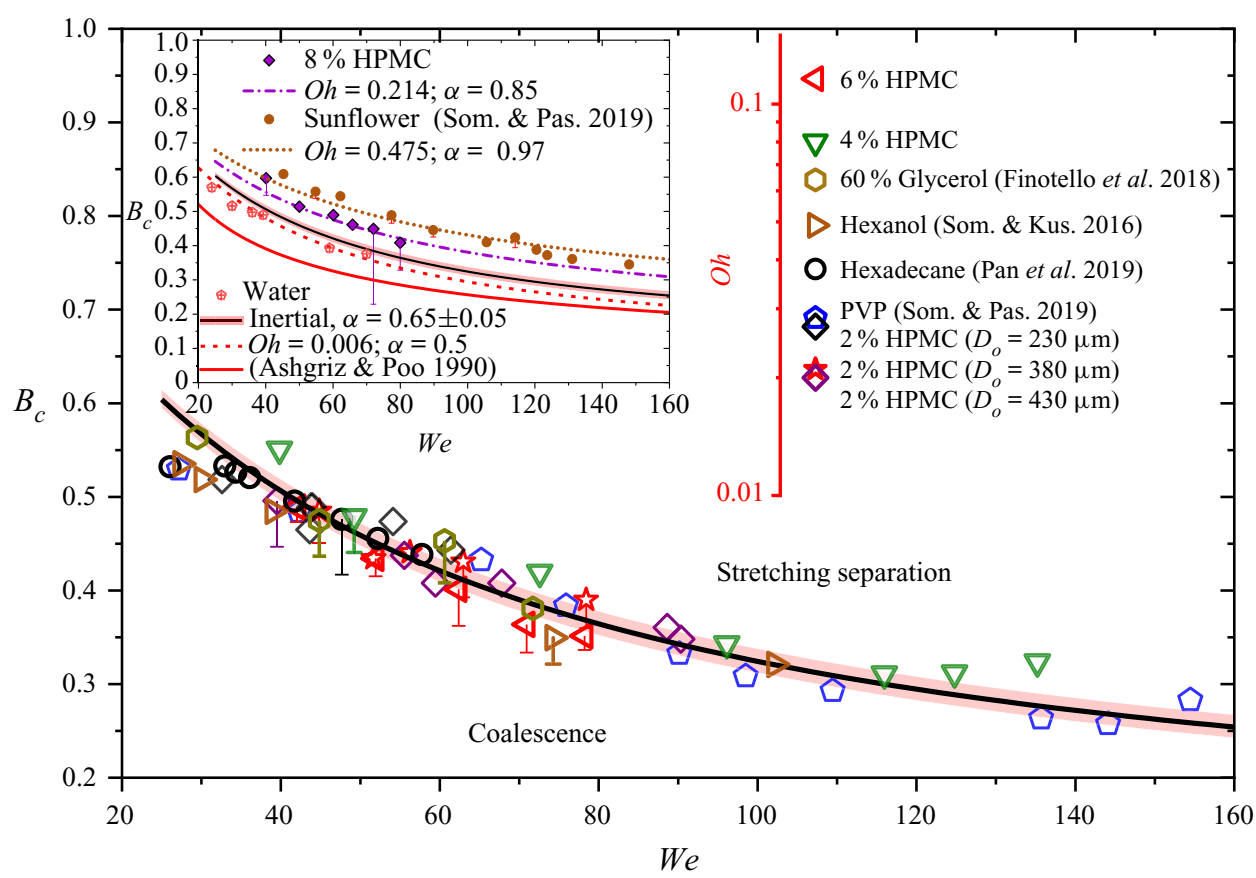

Figure 8. The C-SS transition, demonstrating the inertial behaviour for a wide range of $O h$. The inset image shows the C-SS transition for $O h$ higher and lower than the inertial range. The red Oh scale represents the inertial range $(\alpha=0.65)$. The performance of the model (3.1) is also shown in both figures. The black line represents the model with $\alpha=0.65$, while the red shaded region around it represents the scattering of \pm 0.05 in the plateau of $\alpha(\mathrm{Oh})$. The error bars represent the uncertainty of the transition location.

\subsection{Comparison with the role of $\mathrm{Oh}$ in $\mathrm{RS}$}

Using our findings, we can also shed light on the different roles that $O h$ plays in SS and RS. The role of $O h$ in the latter has been investigated in depth by many authors (e.g. Qian \& Law 1997; Roisman 2004; Roisman et al. 2012; Planchette et al. 2017). Although purely inertial behaviour is seen in the compression phase of head-on collisions (Planchette et al. 2017), increasing $O h$ within this inertial range shifts the RS regime to significantly higher We (from 30 to 190, figure 2 in Huang, Pan \& Josserand 2019). As noted above, such a shift is not seen for the C-SS transition. The difference between these two behaviours can be attributed to viscous loss in the relaxation phase of RS (as the rimmed-lamellar disk retracts and the cylinder forms - see the first column in figure 4 , when $t>0.27 \mathrm{~ms}$ ), which is not present in SS. The viscous loss increases with $\mathrm{Oh}$ and contributes to the total viscous losses that affect the collision outcome (Planchette et al. 2017).

\section{Conclusions}

In this work, we have comprehensively demonstrated the effect of Ohnesorge number on the C-SS transition in binary droplet collisions. Although the literature indicates that increasing viscosity shifts the C-SS transition toward higher Weber numbers, both our experimental and analytical results conclusively show that the C-SS transition is actually inertial for an order-of-magnitude-wide $O h$ range. Surprisingly, a viscous effect exists for $O h$ values both above and below the inertial range identified in this work. We also showed that SS only occurs if the stretched droplets exceed a certain critical dimensionless 


\section{Inertial stretching separation in binary droplet collisions}

length (3.35), which enabled the development of a simple but robust model to capture the $\mathrm{C}-\mathrm{SS}$ transition. The proposed model contains no arbitrarily chosen free parameters; all numerical parameters have been directly specified by readily obtainable experimental data, which is consistent with both our own experiments and existing experimental data found in the literature. Whilst this work is restricted to equal-sized droplet collisions, the effect of size ratio and the three-dimensional evolution on the dynamics would be compelling future work, with the latter potentially enabling an improved mechanistic understanding of our observations to be derived. Nevertheless, our findings have important consequences for applications that rely on targeted collision outcomes, including emulsion fabrication, the dropwise study of chemical reactions and (bio)material printing. Moreover, they are of fundamental importance to other industrial applications such as spray drying, inhaler design, painting, combustion engines, coating and nuclear operations.

Supplementary data. Supplementary material is available at https://doi.org/10.1017/jfm.2021.674.

Funding. This work was supported by the EPSRC project 'Evaporative Drying of Droplets and the Formation of Micro-structured and Functional Particles and Films' (grant no. EP/N025245/1), a Royal Society Enhancement Award (grant no. RGF $\backslash E A \backslash 181002$ ), the University of Leeds and the EPSRC Centre for Doctoral Training in Fluid Dynamics at the University of Leeds (grant no. EP/L01615X/1).

Declaration of interests. The authors report no conflict of interest.

\section{Author ORCIDs.}

(i) Karrar H. Al-Dirawi https://orcid.org/0000-0002-3606-9873;

(1) Khaled H.A. Al-Ghaithi https://orcid.org/0000-0001-8418-4713;

(1) Thomas C. Sykes https://orcid.org/0000-0002-9996-3004;

(ㄱ J. Rafael Castrejón-Pita https://orcid.org/0000-0001-8306-2095;

(1) Andrew E. Bayly https://orcid.org/0000-0001-6354-9015.

\section{REFERENCES}

Al-DiRAWI, K.H. \& BAYly, A.E. 2019 A new model for the bouncing regime boundary in binary droplet collisions. Phys. Fluids 31 (2), 027105.

AL-DiRAWI, K.H. \& BAYLY, A.E. 2020 An experimental study of binary collisions of miscible droplets with non-identical viscosities. Exp. Fluids 61 (2), 50.

Arkhipov, V.A., VAsenin, I.M. \& Trofimov, V.F. 1983 Stability of colliding drops of ideal liquid. J. Appl. Mech. Tech. Phys. 24 (3), 371-373.

Ashgriz, N. \& Poo, J.Y. 1990 Coalescence and separation in binary collisions of liquid drops. J. Fluid Mech. 221, 183-204.

BraZier-Smith, P.R., Jennings, S.G. \& LAtham, J. 1972 The interaction of falling water drops: coalescence. Proc. R. Soc. Lond. A 326 (1566), 393-408.

Castrejón-Pita, A.A., Castrejón-Pita, J.R. \& Hutchings, I.M. 2012 Breakup of liquid filaments. Phys. Rev. Lett. 108 (7), 074506.

Davis, R.D., Jacobs, M.I., Houle, F.A. \& Wilson, K.R. 2017 Colliding-droplet microreactor: rapid on-demand inertial mixing and metal-catalyzed aqueous phase oxidation processes. Anal. Chem. 89 (22), 12494-12501.

Dong, H., CARR, W.W. \& MORRIS, J.F. 2006 An experimental study of drop-on-demand drop formation. Phys. Fluids 18 (7), 072102.

Finotello, G., Kooiman, R.F., Padding, J.T., Buist, K.A., Jongsma, A., Innings, F. \& Kuipers, J.A.M. 2018 The dynamics of milk droplet-droplet collisions. Exp. Fluids 59 (1), 17.

GotaAs, C., Havelka, P., Jakobsen, H.A \& Svendsen, H.F. 2007a Evaluation of the impact parameter in droplet-droplet collision experiments by the aliasing method. Phys. Fluids 19 (10), 102105.

Gotaas, C., Havelka, P., Jakobsen, H.A., Svendsen, H.F., Hase, M., Roth, N. \& Weigand, B. $2007 b$ Effect of viscosity on droplet-droplet collision outcome: experimental study and numerical comparison. Phys. Fluids 19 (10), 102106.

GunN, R. 1965 Collision characteristics of freely falling water drops. Science 150 (3697), 695-701. 


\section{K.H. Al-Dirawi and others}

Henderson, D., Segur, H., Smolka, L.B. \& Wadati, M. 2000 The motion of a falling liquid filament. Phys. Fluids 12 (3), 550-565.

Huang, K.-L., PAn, K.-L. \& Josserand, C. 2019 Pinching dynamics and satellite droplet formation in symmetrical droplet collisions. Phys. Rev. Lett. 123 (23), 234502.

JiAnG, Y.J., UMEMURA, A. \& LAW, C.K. 1992 An experimental investigation on the collision behaviour of hydrocarbon droplets. J. Fluid Mech. 234, 171-190.

Kuschel, M. \& SOMMERfELD, M. 2013 Investigation of droplet collisions for solutions with different solids content. Exp. Fluids 54 (2), 1440.

LEE, J.K., Kim, S., NAM, H.G. \& ZARE, R.N. 2015 Microdroplet fusion mass spectrometry for fast reaction kinetics. Proc. Natl Acad. Sci. USA 112 (13), 3898-3903.

Moretto, L.G., Tso, K., Colonna, N. \& Wozniak, G.J. 1992 New Rayleigh-Taylor-like surface instability and nuclear multifragmentation. Phys. Rev. Lett. 69 (13), 1884.

Pan, K.-L., HuAng, K.-L., Hsieh, W.-T. \& Lu, C.-R. 2019 Rotational separation after temporary coalescence in binary droplet collisions. Phys. Rev. Fluids 4 (12), 123602.

Pan, K.-L., Tseng, Y.-H., Chen, J.-C., Huang, K.-L., Wang, C.-H. \& Lai, M.-C. 2016 Controlling droplet bouncing and coalescence with surfactant. J. Fluid Mech. 799, 603-636.

PARK, R.W. 1970 Behavior of water drops colliding in humid nitrogen. PhD thesis, The University of Wisconsin-Madison, WI.

Planchette, C., Hinterbichler, H., Liu, M., Bothe, D. \& Brenn, G. 2017 Colliding drops as coalescing and fragmenting liquid springs. J. Fluid Mech. 814, 277-300.

Planchette, C., Lorenceau, E. \& Brenn, G. 2010 Liquid encapsulation by binary collisions of immiscible liquid drops. Colloids Surf. A 365 (1-3), 89-94.

Planchette, C., Lorenceau, E. \& Brenn, G. 2012 The onset of fragmentation in binary liquid drop collisions. J. Fluid Mech. 702, 5-25.

QIAN, J. \& LAW, C.K. 1997 Regimes of coalescence and separation in droplet collision. J. Fluid Mech. 331, 59-80.

RAYLEIGH, LORD 1878 On the instability of jets. Proc. Lond. Math. Soc. 1 (1), 4-13.

RoISMAN, I.V. 2004 Dynamics of inertia dominated binary drop collisions. Phys. Fluids 16 (9), 3438-3449.

Roisman, I.V., Planchette, C., Lorenceau, E. \& Brenn, G. 2012 Binary collisions of drops of immiscible liquids. J. Fluid Mech. 690, 512-535.

SAROKA, M.D. \& AShgRIZ, N. 2015 Separation criteria for off-axis binary drop collisions. J. Fluids 2015, 405696.

Sommerfeld, M. \& Kuschel, M. 2016 Modelling droplet collision outcomes for different substances and viscosities. Exp. Fluids 57 (12), 187.

Sommerfeld, M. \& PASternAK, L. 2019 Advances in modelling of binary droplet collision outcomes in sprays: a review of available knowledge. Intl J. Multiphase Flow 117, 182-205.

SuO, S. \& JIA, M. 2020 Correction and improvement of a widely used droplet-droplet collision outcome model. Phys. Fluids 32 (11), 111705.

TANG, C., ZHANG, P. \& LAW, C.K. 2012 Bouncing, coalescence, and separation in head-on collision of unequal-size droplets. Phys. Fluids 24 (2), 022101.

Tsapis, N., Dufresne, E.R., Sinha, S.S., Riera, C.S., Hutchinson, J.W., Mahadevan, L. \& WEITZ, D.A. 2005 Onset of buckling in drying droplets of colloidal suspensions. Phys. Rev. Lett. 94, 018302.

Visser, C.W., KAMPerman, T., KARbAat, L.P., Lohse, D. \& KARPERIEN, M. 2018 In-air microfluidics enables rapid fabrication of emulsions, suspensions, and 3D modular (bio)materials. Sci. Adv. 4 (1), eaao1175.

WANG, C.H., FU, S.Y., KunG, L.J. \& LAw, C.K. 2005 Combustion and microexplosion of collision-merged methanol/alkane droplets. Proc. Combust. Inst. 30 (2), 1965-1972.

Willis, K. \& ORME, M. 2003 Binary droplet collisions in a vacuum environment: an experimental investigation of the role of viscosity. Exp. Fluids 34 (1), 28-41. 